\title{
Structural Balance Control of Complex Dynamical Networks Based on State Observer for Dynamic Connection Relationships
}

\author{
Zilin Gao $\mathbb{D}^{1},{ }^{1}$ Yinhe Wang, ${ }^{2}$ Jiang Xiong, ${ }^{1}$ Yong Pan, ${ }^{1}$ and Yuanyuan Huang $\mathbb{D}^{3}$ \\ ${ }^{1}$ Key Laboratory of Intelligent Information Processing and Control of Chongqing Municipal Institutions of Higher Education, \\ Chongqing Three Gorges University, Chongqing 404100, China \\ ${ }^{2}$ School of Automation, Guangdong University of Technology, Guangzhou 510006, China \\ ${ }^{3}$ School of Computer and Communication Engineering, Changsha University of Science and Technology, Changsha 410016, China \\ Correspondence should be addressed to Zilin Gao; gaozilin321@163.com
}

Received 6 August 2019; Accepted 12 October 2019; Published 11 May 2020

Academic Editor: Mojtaba Ahmadieh Khanesar

Copyright (C) 2020 Zilin Gao et al. This is an open access article distributed under the Creative Commons Attribution License, which permits unrestricted use, distribution, and reproduction in any medium, provided the original work is properly cited.

\begin{abstract}
This paper investigates the observer-based structural balance control for a class of complex dynamical networks. Generally speaking, a complete complex dynamical network is composed of two coupled subsystems, which are called node subsystem (NS) and connection relationship subsystem (CS), respectively. Similar to synchronization and stabilization of networks, the structural balance is another phenomenon of networks and determined by the state of connection relationships. However, it is not feasible to design the controller for the CS directly because the states of the connection relationships are difficult to be measured accurately in practical applications. In order to solve this problem, a state observer for the CS has been designed. Thus, the structural balance controller in the CS can be directly designed by using the estimation information of the state observer. Then, with the help of the Lyapunov stability theory, it is proved that the CS can asymptotically track a given structural balance matrix under the influence of the observer-based controller. Finally, the results derived from this paper are demonstrated by performing a numerical example.
\end{abstract}

\section{Introduction}

In recent decades, complex dynamical networks have drawn considerable attention because many real-world applications need the help of research results about complex dynamical networks, such as neural networks [1], social networks [2], power networks [3], wireless networks [4], and Internet networks [5]. Complex dynamical networks are represented by a group of dynamically interacting nodes with connection relationships between them. That is, the dynamical equations of a complete network mainly contain two parts. The first is the node subsystem (nodes) and the second is the connection relationship subsystem (connection relationships between nodes), where the two subsystems are usually coupled with each other. Moreover, the model of complex dynamical networks helps to understand and examine the dynamical behaviors of networks in a better way. The reason is that some behaviors are reflected by nodes such as synchronization $[6,7]$, stabilization $[8,9]$, and consensus
[10-12], and another characteristic behavior is determined by the connection relationships such as structural balance [13-15].

From the existing research results about the synchronization, stabilization, consensus, and structural balance or other problems of complex dynamical networks, it is assumed that all states in complex dynamical networks, including the states of nodes and connection relationships, can be measured accurately. In fact, due to the large scale of complex dynamical networks (including a large number of nodes and connection relationships) and influence of external environment, technical constraints, and measurement costs, this assumption is too hard to meet for a network [16]. It implies that it is very necessary and important to construct state observers for the complex dynamical networks to estimate the unknown state variables. In $[17,18]$, the state estimation problem is investigated for complex dynamical networks with the coupling time delays. In $[19,20]$, the state estimation problem of complex networks with uncertain 
network parameters and stochastic noisy disturbance is discussed. The papers $[21,22]$ proposed some state estimation methods to solve the problem of packet loss caused by the congestion and transmission error in the networks.

However, the abovementioned papers about the state estimation of complex dynamical networks only discussed the estimation problems of the states in nodes subsystem and assumed that the connection relationships between nodes are known. On the other words, the measurement and state estimation problems of the connection relationships not only are ignored but also they are not concerned with the structural balance control problem of the complex dynamical networks.

Compared to the states of the nodes in the networks, the state values of the connection relationships are more complex and difficult to be measured accurately in practical applications, especially in some real networks such as social networks. Hence, there are only a few papers to study the effective measurement problem of the connection relationships in networks. In [23], a state measurement method of connection relationships is proposed according to the physical interaction between the individuals (nodes). Zeng et al. [24] introduced a measurement method of the social relationships based on adaptive weights. Similar to the state values of the nodes, it is also difficult to obtain all the measured values of the connection relationships, and designing observers to estimate the unmeasured state values of the connection relationships is very necessary. As far as we know, there is only one paper to discuss the state estimation problem of dynamic connection relationships in networks [25]. Unfortunately, this paper only solves the state estimation problem for connection relationships in a class of networks, but does not consider some related control problems, such as structural balance control.

On the other hand, more and more scholars pay attention to the structural balance of networks, and a series of research results have been obtained [13-15, 24, 26-34]. So far, the models of structural balance have gone through the following stages. (i) The signed network model, in which the state values of connection strength are expressed by only using integers 0 and $\pm 1[24,26]$; (ii) the time continuous models, which are described by a set of differential equations or a matrix differential equation $[13,15,27-32]$, but they did not consider the effects of internal state motions of the nodes on the evolution of connection relationships; (iii) the model is described by a Riccati dynamical equation with the coupling matrix about the internal state of nodes [14, 33, 34], which implies that any dynamical changes of nodes (or connection relationships) will cause the connection relationships (or nodes) to be changed via the effective coupling. These papers investigate the time evolution behaviors of the connection relationships and show the structural balance of networks. However, considering that the state values of connection relationships cannot be measured accurately; thus, there are only a few papers to discuss the structural balance control problems of networks. In $[15,31]$, the structural balance of networks is realized by changing the values of the connection relationships between the nodes and a "reference" agent (node). The papers [14, 34] proposed a complex dynamical network model composed of the node subsystem and connection relationship subsystem, where the two subsystems are coupled with each other. Thus, the structural balance of networks can be achieved by designing the controller in node subsystem, which can control the states of nodes to force the connection relationship subsystem to be structural balance via the effective coupling. To the best of our knowledge, the structural balance control of networks based on the state observer of connection relationships has not been studied.

Inspired by the above discussions, we mainly focus on the design of the structural balance controller with the estimation information of the state observer for the connection relationships subsystem in this paper. Firstly, we proposed a complex dynamical network model, which is composed of the nodes subsystem and connection relationships subsystem with coupled each other. Then, we designed a state observer for the connection relationship subsystem. Finally, the controller in connection relationships subsystem has been proposed by using the estimation information of the state observer, which can guarantee the phenomenon of structural balance emerges in the network. That is, the estimation and direct control problems of the connection relationships subsystem are solved in this paper.

This paper is organized as the following sections. Section 2 proposes a complex dynamical network model, which is composed of the nodes subsystem and the connection relationships subsystem with outputs. In Section 3, the state observer for the connection relationships subsystem is designed. Section 4 completes the design of the structural balance controller in connection relationship subsystem by using the estimation information of the state observer. The simulation example is presented in Section 5. Finally, the conclusions are given in Section 6.

\section{Network Model Description}

In this paper, we consider a class of the undirected complex dynamical network with $N$ nodes, which are composed of the node subsystem and the connection relationship subsystem. If each node is $n$-dimensional continuous-time system, then the node subsystem and the connection relationship subsystem can be described as follows (see $[14,33,34])$ :

$$
\begin{aligned}
& \dot{x}_{i}=f_{i}\left(x_{i}\right)+c_{i} \sum_{j=1}^{N} p_{i j}(t) H_{j}\left(x_{j}\right), \quad i=1,2, \ldots, N, \\
& \left\{\begin{array}{l}
\dot{P}=\Theta P+P \Theta^{T}+U+U^{T}+G(x)+G(x)^{T}, \\
Y=\Upsilon P,
\end{array}\right.
\end{aligned}
$$

where $x_{i}=\left[\begin{array}{llll}x_{i 1} & x_{i 2} & \ldots & x_{i n}\end{array}\right]^{T} \in R^{n}$ is the state vector of node $i$; the vector functions $f_{i}\left(x_{i}\right)=\left[f_{i 1}\left(x_{i}\right) f_{i 2}\left(x_{i}\right) \ldots\right.$ $\left.f_{\text {in }}\left(x_{i}\right)\right]^{T}$ and $H_{j}\left(x_{j}\right)=\left[\begin{array}{lllll}H_{j 1}\left(x_{j}\right) & H_{j 2}\left(x_{j}\right) & \ldots & H_{j n}\left(x_{j}\right)\end{array}\right]^{T}$; $c_{i}$ is a given positive constant, and it represents the common connection strength of the $i$ th node in the networks; the matrix $\Theta \in R^{N \times N}$ is a constant matrix; $G(x) \in R^{N \times N}$ represents the coupling matrix with the internal state of the nodes, and 
$x=\left[\begin{array}{llll}x_{1}^{T} & x_{2}^{T} & \ldots & x_{N}^{T}\end{array}\right]^{T} \in R^{N n} ; \quad U \in R^{N \times N}$ is the control input; $\Upsilon \in R^{N_{1} \times N}$ is the output matrix of the connection relationship subsystem (2); and the connection relationships matrix $P=P(t)=\left[p_{i j}(t)\right]_{N \times N}$, where the time-varying function $p_{i j}(t)$ expresses the weighted value of connection relationship between the node $i$ and the node $j$ in the network and $p_{j i}=p_{i j}$ for undirected networks, especially when $i=j$, $p_{i j}$ indicates the relationship strength of the node itself, $i=1,2, \ldots, N$.

Now, we introduce the definitions of $\operatorname{vec}(\cdot)$ operator and the Kronecker product of matrices as follows.

Definition 1 (see [35]). The application vec: $R^{k \times l} \longrightarrow R^{k l}$, defined by

$$
\operatorname{vec}(H)=\left[h_{11}, \ldots, h_{1 l}, h_{21}, \ldots, h_{2 l}, \ldots, h_{k 1}, \ldots, h_{k l}\right]^{T} \text {, }
$$

where $H=\left[h_{i j}\right]_{k \times l} \in R^{k \times l}$ is called the vectorization operator.

Definition 2 (see [35]). If $H \in R^{k \times l}$ and $Z \in R^{c \times d}$, then the Kronecker product of $H$ and $Z$, denoted as $H \otimes Z \in R^{k c \times l d}$, is defined by the following matrix:

$$
H \otimes Z=\left(\begin{array}{cccc}
h_{11} Z & h_{12} Z & \cdots & h_{1 l} Z \\
h_{21} Z & h_{22} Z & \cdots & h_{2 l} Z \\
\cdot & \cdot & \cdot & \cdot \\
\cdot & \cdot & \cdot & \cdot \\
h_{k 1} Z & h_{k 2} Z & \cdots & h_{k l} Z
\end{array}\right)
$$

Then, the following basic properties of the vectorization operator $\operatorname{vec}(\cdot)$ and Kronecker product can be obtained from [35].

$$
\begin{aligned}
& \text { (1) }(H \otimes X)(W \otimes D)=(H W) \otimes(X D) \\
& \text { (2) }(H \otimes D)^{T}=H^{T} \otimes D^{T} \\
& \text { (3) }(X \otimes W)^{-1}=X^{-1} \otimes W^{-1} \\
& \text { (4) } \operatorname{vec}(H X Z)=\left(H \otimes Z^{T}\right) \operatorname{vec}(X) \\
& \text { (5) } \operatorname{vec}(H X+X Z)=\left(H \otimes I+I \otimes Z^{T}\right) \operatorname{vec}(X)
\end{aligned}
$$

where $X$ and $W$ are matrices with compatible dimensions and $I$ represents the identity matrix with compatible dimensions. Especially, it is assumed that both $X$ and $W$ are invertible in property (3).

According to (3), (4), and the Kronecker product properties, the Riccati dynamical equation (2) can be rewritten as follows:

$$
\left\{\begin{array}{l}
\operatorname{vec}(\dot{P})=A \operatorname{vec}(P)+\operatorname{vec}(U)+\operatorname{vec}\left(U^{T}\right)+\operatorname{vec}(G(x)) \\
\quad+\operatorname{vec}\left(G(x)^{T}\right) \\
\operatorname{vec}(Y)=C_{1} \operatorname{vec}(P)
\end{array}\right.
$$

where $A=\Theta \otimes I_{N}+I_{N} \otimes \Theta, C_{1}=\Upsilon \otimes I_{N}$, and $I_{N}$ denotes the identity matrix of order $N$.

Assumption 1. For the connection relationships subsystem (2), we assume that the double matrix $(\Theta, \Upsilon)$ is completely stable.

If Assumption 1 holds, then we can obtain a matrix $K \in R^{N \times N_{1}}$, which can make $\Theta+K \Upsilon$ to be a Hurwitz stable matrix. Thus, as long as any matrix $Q>0$ is given, there must be one and only one positive definite matrix $M \in R^{N \times N}$ that satisfies the following Lyapunov equation:

$$
(\Theta+K \Upsilon)^{T} M+M(\Theta+K \Upsilon)=-Q .
$$

Lemma 1. If Assumption 1 is true, then the following Lyapunov equations are obtained:

$$
\begin{aligned}
& \left(\Theta \otimes \mathrm{I}_{N}+K_{1} C_{1}\right)^{T} \tilde{M}+\tilde{M}\left(\Theta \otimes \mathrm{I}_{N}+K_{1} C_{1}\right)=-Q_{1}, \\
& \left(I_{N} \otimes \Theta+K_{2} C_{2}\right)^{T} \tilde{M}+\tilde{M}\left(\mathrm{I}_{N} \otimes \Theta+K_{2} C_{2}\right)=-Q_{2},
\end{aligned}
$$

where $\tilde{M}=M \otimes M, Q_{1}=M \otimes Q, Q_{2}=Q \otimes M, K_{1}=K \otimes I_{N}$, $K_{2}=I_{N} \otimes K$, and $C_{2}=I_{N} \otimes Y$. Clearly, $\tilde{M}>0, Q_{1}>0$, and $Q_{2}>0$.

Proof. If Assumption 1 is true, then we can obtain (6), by which the following equations can be obtained:

$$
\begin{gathered}
{\left[(\Theta+K \Upsilon)^{T} M\right] \otimes I_{N}+[M(\Theta+K \Upsilon)] \otimes I_{N}=-Q \otimes I_{N},} \\
I_{N} \otimes\left[(\Theta+K \Upsilon)^{T} M\right]+I_{N} \otimes[M(\Theta+K Y)]=-I_{N} \otimes Q .
\end{gathered}
$$

Using the properties of Kronecker product, (8a) and (8b) can be rewritten as

$$
\begin{gathered}
{\left[\Theta \otimes I_{N}+\left(K \otimes I_{N}\right)\left(\Upsilon \otimes I_{N}\right)\right]^{T}\left(M \otimes I_{N}\right)+\left(M \otimes I_{N}\right)} \\
\cdot\left[\Theta \otimes I_{N}+\left(K \otimes I_{N}\right)\left(\Upsilon \otimes I_{N}\right)\right]=-Q \otimes I_{N}, \\
{\left[I_{N} \otimes \Theta+\left(I_{N} \otimes K\right)\left(I_{N} \otimes \Upsilon\right)\right]^{T}\left(I_{N} \otimes M\right)+\left(I_{N} \otimes M\right)} \\
\cdot\left[I_{N} \otimes \Theta+\left(I_{N} \otimes K\right)\left(I_{N} \otimes \Upsilon\right)\right]=-I_{N} \otimes Q .
\end{gathered}
$$

Thus, we can obtain

$$
\begin{aligned}
& {\left[\Theta \otimes I_{N}+K_{1} C_{1}\right]^{T}\left(M \otimes I_{N}\right)+\left(M \otimes I_{N}\right)\left[\Theta \otimes I_{N}+K_{1} C_{1}\right]} \\
& \quad=-Q \otimes I_{N}, \\
& {\left[I_{N} \otimes \Theta+K_{2} C_{2}\right]^{T}\left(I_{N} \otimes M\right)+\left(I_{N} \otimes M\right)\left[I_{N} \otimes \Theta+K_{2} C_{2}\right]} \\
& \quad=-I_{N} \otimes Q .
\end{aligned}
$$


If both sides of equalities (10a) and (10b) are multiplied by $\left(I_{N} \otimes M\right)$ and $\left(M \otimes I_{N}\right)$ from right, respectively, then we can obtain

$$
\begin{gathered}
{\left[\Theta \otimes I_{N}+K_{1} C_{1}\right]^{T}\left(M \otimes I_{N}\right)\left(I_{N} \otimes M\right)+\left(M \otimes I_{N}\right)} \\
\cdot\left[\Theta \otimes I_{N}+K_{1} C_{1}\right]\left(I_{N} \otimes M\right)=-\left(Q \otimes I_{N}\right)\left(I_{N} \otimes M\right), \\
{\left[I_{N} \otimes \Theta+K_{2} C_{2}\right]^{T}\left(I_{N} \otimes M\right)\left(M \otimes I_{N}\right)+\left(I_{N} \otimes M\right)} \\
\cdot\left[I_{N} \otimes \Theta+K_{2} C_{2}\right]\left(M \otimes I_{N}\right)=-\left(I_{N} \otimes Q\right)\left(M \otimes I_{N}\right) .
\end{gathered}
$$

It is noticed that $\left(I_{N} \otimes M\right)\left(M \otimes I_{N}\right)=M \otimes M=$ $\left(M \otimes I_{N}\right)\left(I_{N} \otimes M\right)$. Therefore, equalities (11a) and (11b) can be rewritten as follows:

$$
\begin{aligned}
& {\left[\Theta \otimes I_{N}+K_{1} C_{1}\right]^{T}(M \otimes M)+(M \otimes M)\left[\Theta \otimes I_{N}+K_{1} C_{1}\right]} \\
& \quad=-Q \otimes M, \\
& {\left[I_{N} \otimes \Theta+K_{2} C_{2}\right]^{T}(M \otimes M)+(M \otimes M)\left[I_{N} \otimes \Theta+K_{2} C_{2}\right]} \\
& \quad=-M \otimes Q .
\end{aligned}
$$

This completes the proof of Lemma 1.

Assumption 2. The coupling matrix $G(x)$ in subsystem (2) satisfies $G(x)=M^{-1} \Upsilon^{T} \Psi(x) M^{-1}$, where $\Psi(x) \in R^{N_{1} \times N}$ and $\|\Psi(x)\| \leq \rho(t)\|x\|^{2}, \rho(t)>0$.

\section{The State Observer Design}

In this paper, the state $P$ in subsystem (2) cannot be measured accurately, which implies that the states of structural balance controller $U$ cannot contain $P$ directly. Therefore, it is necessary to design an observer to estimate the state $P$ in (2), and the definition of the state observer about subsystem (2) is given as follows.

Definition 3. For a given matrix dynamical system $\dot{\hat{P}}=\mathbb{F}(\widehat{P}, Y, x, \rho)$, if the state of node subsystem (1) can be measured accurately and $P-\widehat{P} \stackrel{\leftrightarrow}{\longrightarrow} 0$ holds, then the matrix dynamical system $\widehat{\widehat{P}}=\mathbb{F}(\widehat{P}, Y, x, \rho)$ is called a state observer of subsystem (2).

If Assumptions 1 and 2 are true, we can design the state observer of subsystem (2) as follows:

$$
\begin{aligned}
\dot{\widehat{P}}= & (\Theta+K \Upsilon) \widehat{P}+\widehat{P}(\Theta+K \Upsilon)^{T}+U+U^{T}+L(\widehat{P}, Y, x, \rho) \\
& -K Y-Y^{T} K^{T} .
\end{aligned}
$$

Using vec $(\cdot)$ operator for (13), the following equation can be obtained:

$$
\begin{aligned}
\operatorname{vec}(\dot{\widehat{P}})= & \left(A+K_{1} C_{1}+K_{2} C_{2}\right) \operatorname{vec}(\widehat{P})+\operatorname{vec}(U)+\operatorname{vec}\left(U^{T}\right) \\
& +\operatorname{vec}(L(\widehat{P}, Y, x, \rho))-K_{1} \operatorname{vec}(Y)-K_{2} \operatorname{vec}\left(Y^{T}\right),
\end{aligned}
$$

where $\widehat{P}$ represents the estimated value of the state $P$ in subsystem (2), and the robust term $L(\widehat{P}, Y, x, \rho)=$ $\left\{\begin{array}{ll}\Omega+\Omega^{T}, & \Upsilon \widehat{P} \neq Y \\ 0, & \Upsilon \widehat{P}=Y\end{array}\right.$, where $\Omega=\rho(t)\|x\|^{2}\left(M^{-1} \Upsilon^{T}(Y-\Upsilon \widehat{P})\right.$ $\left.M^{-1} /\|Y-\Upsilon \widehat{P}\|\right) . \quad$ Clearly, $\quad \operatorname{vec}(L(\widehat{P}, Y, x, \rho))=$ $\left\{\begin{array}{ll}\operatorname{vec}(\Omega)+\operatorname{vec}\left(\Omega^{T}\right), & C_{1} \operatorname{vec}(\widehat{P}) \neq \operatorname{vec}(Y) \\ 0, & C_{1} \operatorname{vec}(\widehat{P})=\operatorname{vec}(Y)\end{array}\right.$. At the same time, we note that the equality $\|Y-\Upsilon \widehat{P}\|=\left\|Y^{T}-\widehat{P} \Upsilon^{T}\right\|=$ $\|\operatorname{vec}(Y-\Upsilon \widehat{P})\|=\left\|\operatorname{vec}\left(Y^{T}-\widehat{P} \Upsilon^{T}\right)\right\| \quad$ holds, then $\operatorname{vec}(\Omega)=\rho(t)\|x\|^{2}\left(\tilde{M}^{-1} C_{1}^{T}\left[\operatorname{vec}(Y)-C_{1} \operatorname{vec}(\widehat{P})\right] / \| \operatorname{vec}(Y)-\right.$ $\left.C_{1} \operatorname{vec}(\widehat{P}) \|\right)$ and $\operatorname{vec}\left(\Omega^{T}\right)=\rho(t)\|x\|^{2}\left(\tilde{M}^{-1} C_{2}^{T}\left[\operatorname{vec}\left(Y^{T}\right)-\right.\right.$ $\left.\left.C_{2} \operatorname{vec}(\widehat{P})\right] /\left\|\operatorname{vec}\left(Y^{T}\right)-C_{2} \operatorname{vec}(\widehat{P})\right\|\right)$ can be obtained.

In this paper, the estimation error is denoted by $E=P-\widehat{P}$. By using properties of $\operatorname{vec}(\cdot)$ operator and Kronecker product, the following error system can be obtained:

$$
\begin{aligned}
\operatorname{vec}(\dot{E})= & A \operatorname{vec}(P)-\left[\left(A+K_{1} C_{1}+K_{2} C_{2}\right) \operatorname{vec}(\widehat{P})\right. \\
& \left.+\operatorname{vec} L(\widehat{P}, Y, x, \rho)-K_{1} \operatorname{vec}(Y)-K_{2} \operatorname{vec}\left(Y^{T}\right)\right] \\
= & \left(A+K_{1} C_{1}+K_{2} C_{2}\right) \operatorname{vec}(E)+\widetilde{M}^{-1} C_{1}^{T} \operatorname{vec}(\Psi(x)) \\
& +\tilde{M}^{-1} C_{2}^{T} \operatorname{vec}\left(\Psi(x)^{T}\right)-\operatorname{vec} L(\widehat{P}, Y, x, \rho) .
\end{aligned}
$$

Lemma 2. If Assumptions 1 and 2 are true, then the matrix dynamical system (13) is the state observer of subsystem (2).

Proof. Consider the following positive definite function:

$$
V_{1}=\frac{1}{2} \operatorname{vec}(E)^{T} \tilde{M} \operatorname{vec}(E) \text {. }
$$

Then, the orbit derivative of $V$ along (16) reads as 


$$
\begin{aligned}
& \dot{V}_{1}=\operatorname{vec}(E)^{T} \tilde{M} \operatorname{vec}(\dot{E}) \\
& =\operatorname{vec}(E)^{T} \tilde{M}\left\{\left(A+K_{1} C_{1}+K_{2} C_{2}\right) \operatorname{vec}(E)+\tilde{M}^{-1} C_{1}^{T} \operatorname{vec}(\Psi(x))+\tilde{M}^{-1} C_{2}^{T} \operatorname{vec}\left(\Psi(x)^{T}\right)-\operatorname{vec} L(\widehat{P}, Y, x)\right\} \\
& =\operatorname{vec}(E)^{T} \tilde{M}\left(\Theta \otimes \mathrm{I}_{N}+K_{1} C_{1}\right) \operatorname{vec}(E)+\operatorname{vec}(E)^{T} \tilde{M}\left(\mathrm{I}_{N} \otimes \Theta+K_{2} C_{2}\right) \operatorname{vec}(E) \\
& +\operatorname{vec}(E)^{T} \widetilde{M}\left[\widetilde{M}^{-1} C_{1}^{T} \operatorname{vec}(\Psi(x))+\widetilde{M}^{-1} C_{2}^{T} \operatorname{vec}\left(\Psi(x)^{T}\right)-\operatorname{vec} L(\widehat{P}, Y, x)\right] \\
& =\frac{1}{2} \operatorname{vec}(E)^{T}\left[\left(\Theta \otimes \mathrm{I}_{N}+K_{1} C_{1}\right)^{T} \tilde{M}+\tilde{M}\left(\Theta \otimes \mathrm{I}_{N}+K_{1} C_{1}\right)\right] \operatorname{vec}(E) \\
& +\frac{1}{2} \operatorname{vec}(E)^{T}\left[\left(\mathrm{I}_{N} \otimes \Theta+K_{2} C_{2}\right)^{T} \tilde{M}+\tilde{M}\left(\mathrm{I}_{N} \otimes \Theta+K_{2} C_{2}\right)\right] \operatorname{vec}(E) \\
& +\operatorname{vec}(E)^{T} \widetilde{M}\left[\tilde{M}^{-1} C_{1}^{T} \operatorname{vec}(\Psi(x))+\tilde{M}^{-1} C_{2}^{T} \operatorname{vec}\left(\Psi(x)^{T}\right)-\operatorname{vec} L(\widehat{P}, Y, x)\right] \\
& =-\frac{1}{2} \operatorname{vec}(E)^{T} Q_{1} \operatorname{vec}(E)-\frac{1}{2} \operatorname{vec}(E)^{T} Q_{2} \operatorname{vec}(E) \\
& +\operatorname{vec}(E)^{T} C_{1}^{T} \operatorname{vec}(\Psi(x))+\operatorname{vec}(E)^{T} C_{2}^{T} \operatorname{vec}\left(\Psi(x)^{T}\right) \\
& -\operatorname{vec}(E)^{T} \tilde{M} \begin{cases}\operatorname{vec}(\Omega)+\operatorname{vec}\left(\Omega^{T}\right), & C_{1} \operatorname{vec}(\widehat{P}) \neq \operatorname{vec}(Y) \\
0, & C_{1} \operatorname{vec}(\widehat{P})=\operatorname{vec}(Y)\end{cases} \\
& \leq-\frac{1}{2} \operatorname{vec}(E)^{T} Q_{1} \operatorname{vec}(E)-\frac{1}{2} \operatorname{vec}(E)^{T} Q_{2} \operatorname{vec}(E) \\
& +\left\|\operatorname{vec}(E)^{T} C_{1}^{T}\right\|\|\operatorname{vec}(\Psi(x))\|+\left\|\operatorname{vec}(E)^{T} C_{2}^{T}\right\|\left\|\operatorname{vec}\left(\Psi(x)^{T}\right)\right\| \\
& - \begin{cases}\operatorname{vec}(E)^{T} \widetilde{M} \operatorname{vec}(\Omega)+\operatorname{vec}(E)^{T} \widetilde{M} \operatorname{vec}\left(\Omega^{T}\right), & C_{1} \operatorname{vec}(\widehat{P}) \neq \operatorname{vec}(Y) \\
0, & C_{1} \operatorname{vec}(\widehat{P})=\operatorname{vec}(Y)\end{cases} \\
& \leq-\frac{1}{2} \operatorname{vec}(E)^{T} Q_{1} \operatorname{vec}(E)-\frac{1}{2} \operatorname{vec}(E)^{T} Q_{2} \operatorname{vec}(E)+\rho(t)\|x\|^{2}\left\|\operatorname{vec}(E)^{T} C_{1}^{T}\right\|+\rho(t)\|x\|^{2}\left\|\operatorname{vec}(E)^{T} C_{2}^{T}\right\| \\
& - \begin{cases}\rho(t)\|x\|^{2}\left(\left\|C_{1} \operatorname{vec}(E)\right\|+\left\|C_{2} \operatorname{vec}(E)\right\|\right), & C_{1} \operatorname{vec}(\widehat{P}) \neq \operatorname{vec}(Y) \\
0, & C_{1} \operatorname{vec}(\widehat{P})=\operatorname{vec}(Y)\end{cases} \\
& =-\frac{1}{2} \operatorname{vec}(E)^{T} Q_{1} \operatorname{vec}(E)-\frac{1}{2} \operatorname{vec}(E)^{T} Q_{2} \operatorname{vec}(E) \leq 0 .
\end{aligned}
$$

From inequality (17), we can obtain that the estimation

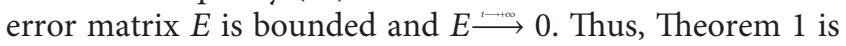
proved.

\section{The Structural Balance Controller Design}

Although we cannot directly use state $P$ in subsystem (2) to design the structural balance controller $U$, but the state of subsystem (1), the state estimation information $\widehat{P}$ in state observer (13) and the output $Y$ in subsystem (2) can be used in the structural balance controller $U$.

Firstly, some useful definitions about structural balance are given as follows.
Definition 4. A symmetric matrix $S=\left[s_{\alpha \beta}\right] \in R^{m \times m}$ is structurally balanced if the inequality $s_{\alpha \beta} s_{\beta \rho} s_{\rho \alpha}>0$ holds, where $\alpha, \beta, \rho=1,2, \ldots, m$ and $m \geq 3$.

According to Definition 4, the asymptotic structural balance of complex dynamical networks can be defined as follows.

Definition 5. Consider the complex dynamical network, which is composed of subsystems (1) and (2). For a given structural balance matrix $P^{*}$, if subsystem (2) can asymptotically track the matrix $P^{*}$ under the influences of controller $U$, i.e., $P(t) \stackrel{\longrightarrow \rightarrow \infty}{\longrightarrow} P^{*}$, then this complex dynamical network is called as structural balance. 
Therefore, the control objective in this paper is given as follows.

Control objective: for the complex dynamical network, which consists of subsystems (1) and (2), we need try to design the controller $U(\widehat{P}, Y, x, \rho)$ in subsystem (2), such that the phenomenon of structural balance emerges in this complex dynamical network.

In order to achieve the above control objective, the following control scheme is proposed for subsystem (2):

$$
U=-(\Theta+\mathrm{K} Y) P^{*}+\mathrm{K} Y+\Lambda,
$$

where $\Lambda= \begin{cases}-\rho(t)\|x\|\left(\tilde{M}^{-1} \Upsilon^{T}(Y-\Upsilon \widehat{P}) \tilde{M}^{-1} /\left\|\operatorname{vec}(Y)-C_{1} \operatorname{vec}(\widehat{P})\right\|\right), & Y \neq \Upsilon \widehat{P} \\ 0, & Y=\Upsilon \widehat{P}\end{cases}$ it is easy to see that $L(\widehat{P}, Y, x, \rho)=-\Lambda-\Lambda^{T}$.

According to (3), (4) and the properties of vec(.) operator and Kronecker product, equation (18) can be rewritten as

$$
\operatorname{vec}(U)=-\left(\Theta \otimes I_{N}+K_{1} C_{1}\right) \operatorname{vec}\left(P^{*}\right)+K_{1} \operatorname{vec}(Y)+\operatorname{vec}(\Lambda),
$$

$\operatorname{vec}\left(U^{T}\right)=-\left(I_{N} \otimes \Theta+K_{2} C_{2}\right) \operatorname{vec}\left(P^{*}\right)+K_{2} \operatorname{vec}\left(Y^{T}\right)+\operatorname{vec}\left(\Lambda^{T}\right)$.

Let $\bar{P}=\widehat{P}-P^{*}$, we can deduce from (14) that

$$
\begin{aligned}
\operatorname{vec}(\dot{\bar{P}})= & \left(A+K_{1} C_{1}+K_{2} C_{2}\right) \operatorname{vec}\left(\bar{P}+P^{*}\right)+\operatorname{vec}(U) \\
& +\operatorname{vec}\left(U^{T}\right)+\operatorname{vec}(L(\widehat{P}, Y, x, \rho))-K_{1} \operatorname{vec}(Y) \\
& -K_{2} \operatorname{vec}\left(Y^{T}\right) .
\end{aligned}
$$

Lemma 3. The estimation state $\widehat{P}$ in observer (13) can asymptotically track the structural balance matrix $P^{*}$ by using controller (18).

Proof. The following equation can be obtained from Lemma 1:

$$
\begin{aligned}
(A & \left.+K_{1} C_{1}+K_{2} C_{2}\right)^{T} \tilde{M}+\tilde{M}\left(A+K_{1} C_{1}+K_{2} C_{2}\right) \\
& =-\left(Q_{1}+Q_{2}\right) .
\end{aligned}
$$

Then, the following positive definite function is considered for error system (21):

$$
V_{2}=\frac{1}{2} \operatorname{vec}(\bar{P})^{T} \tilde{M} \operatorname{vec}(\bar{P}) .
$$

Calculating the time derivative of $V_{2}$ along (21) gives that

$$
\begin{aligned}
& \dot{V}_{2}=\operatorname{vec}(\bar{P})^{T} \tilde{M} \operatorname{vec}(\dot{\bar{P}}) \\
& =\operatorname{vec}(\bar{P})^{T} \tilde{M}\left[\left(A+K_{1} C_{1}+K_{2} C_{2}\right) \operatorname{vec}\left(\bar{P}+P^{*}\right)+\operatorname{vec}(U)\right. \\
& +\operatorname{vec}\left(U^{T}\right)+\operatorname{vec}(L(\widehat{P}, Y, x, \rho))-K_{1} \operatorname{vec}(Y) \\
& \left.-K_{2} \operatorname{vec}\left(Y^{T}\right)\right] \\
& =\operatorname{vec}(\bar{P})^{T} \tilde{M}\left(A+K_{1} C_{1}+K_{2} C_{2}\right) \operatorname{vec}(\bar{P}) \\
& +\operatorname{vec}(\bar{P})^{T} \tilde{M}\left[\left(A+K_{1} C_{1}+K_{2} C_{2}\right) \operatorname{vec}\left(P^{*}\right)\right. \\
& -\left(\Theta \otimes I_{N}+K_{1} C_{1}\right) \operatorname{vec}\left(P^{*}\right)+K_{1} \operatorname{vec}(Y)+\operatorname{vec}(\Lambda) \\
& -\left(I_{N} \otimes \Theta+K_{2} C_{2}\right) \operatorname{vec}\left(P^{*}\right)+K_{2} \operatorname{vec}\left(Y^{T}\right)+\operatorname{vec}\left(\Lambda^{T}\right) \\
& \left.+\operatorname{vec}(L(\widehat{P}, Y, x, \rho))-K_{1} \operatorname{vec}(Y)-K_{2} \operatorname{vec}\left(Y^{T}\right)\right] \\
& =\operatorname{vec}(\bar{P})^{T} \tilde{M}\left(A+K_{1} C_{1}+K_{2} C_{2}\right) \operatorname{vec}(\bar{P}) \\
& =\frac{1}{2} \operatorname{vec}(\bar{P})^{T}\left[\tilde{M}\left(A+K_{1} C_{1}+K_{2} C_{2}\right)\right. \\
& \left.+\left(A+K_{1} C_{1}+K_{2} C_{2}\right)^{T} \tilde{M}\right] \operatorname{vec}(\bar{P}) \\
& =-\frac{1}{2} \operatorname{vec}(\bar{P})^{T}\left(Q_{1}+Q_{2}\right) \operatorname{vec}(\bar{P})<0 .
\end{aligned}
$$

The inequality (24) shows that $\operatorname{vec}(\bar{P}) \stackrel{\leftrightarrow \infty}{\longrightarrow} 0$. Thus, Lemma 3 is completely proved.

Theorem 1. For the complex dynamical network composed of subsystems (1) and (2), if Assumptions 1 and 2 are true, then the state $P$ in subsystem (2) can asymptotically track a given structural balance matrix $P^{*}$ by the effect of controller (18), such that the structural balance of this network is achieved.

Proof. From Lemmas 2 and 3, we can obtain that $\lim _{t \rightarrow \infty}[P(t)-\widehat{P}(t)]=0$ and $\lim _{t \longrightarrow \infty}\left[\widehat{P}(t)-P^{*}\right]=0$, respectively. Therefore, it is not difficult to get that $\lim _{t \rightarrow \infty}[P(t)-$ $\left.\widehat{P}(t)+\lim _{t \rightarrow \infty} \widehat{P}(t)-P^{*}\right]=\lim _{t \rightarrow \infty}\left[P(t)-P^{*}\right]=0$, this result proves Theorem 1 is true. 


\section{Simulation Example}

In this paper, a continuous analog Hopfield network [36] is considered, which is composed of 10 neurons (nodes), and the $i$ th neuron can be described as

$$
\dot{x}_{i}=-\frac{1}{\tau_{i}} x_{i}+\frac{1}{C_{i}} I_{i}+\frac{1}{C_{i}} \sum_{j=1}^{N} p_{i j} H_{j}\left(x_{j}\right)
$$

where $x_{i} \in R$ is the state of the $i$ th neuron; $C_{i}$ expresses the input capacitance of the $i$ th neuron; the decay term $\tau_{i}=R_{i} C_{i}$ is a positive constant, in which $R_{i}$ is the effective resistance of the $i$ th neuron; $I_{i}$ is the input current from other neurons external to the circuit; $p_{i j}=p_{j i}$ represents the strength of synapse between the $i$ th and the $j$ th neuron; and the coupling function $H_{j}\left(x_{j}\right)=\left(1-e^{-x_{j}}\right) /\left(1+e^{-x_{j}}\right)$. Thus, network (25) can be described by the nodes subsystem (1) if we choose $f_{i}\left(x_{i}\right)=-\left(1 / \tau_{i}\right) x_{i}+\left(1 / C_{i}\right) I_{i}$ and $c_{i}=\left(1 / C_{i}\right)$.

Then, we assume that the changes of the strength $p_{i j}$ satisfy the Riccati differential equation (2), and choose $\Psi(x)=\left[\begin{array}{cccc}x_{1}^{T} x_{1} & x_{1}^{T} x_{2} & \cdots & x_{1}^{T} x_{10} \\ x_{2}^{T} x_{1} & x_{2}^{T} x_{2} & \cdots & x_{2}^{T} x_{10} \\ \vdots & \vdots & \ddots & \vdots \\ x_{N_{1}}^{T} x_{1} & x_{N_{1}}^{T} x_{2} & \cdots & x_{N_{1}}^{T} x_{10}\end{array}\right], \quad i, j=1,2, \ldots, 10 . \quad$ If we choose $N_{1}=5$, then the matrices $\Theta \in R^{10 \times 10}$ and $\Upsilon \in R^{5 \times 10}$ in (2) can be generated by the following rules in Matlab:

(i) Each element in matrices $\Theta^{*} \in R^{10 \times 10}$ and $\Upsilon^{*} \in R^{5 \times 10}$ is a randomly generated integer in range $[-1,1]$.

(ii) Let $\Theta=a \cdot \Theta^{*}$ and $\Upsilon=a \cdot \Upsilon^{*}$, where $a$ is a known positive constant.

(iii) If the matrices $\Theta$ and $\Upsilon$ generated in Step (ii) satisfy Assumption 1, proceed to the next step or repeat Step (i).

(iv) Each element in column vector $z \in R^{10}$ is +1 or -1 , which is randomly generated. Then, we can choose $P^{*}=b \cdot z z^{T}$, where $b>0$. In this paper, we choose $a=0.025, b=5$ in the process of simulation.

Therefore, we can obtain the matrices $K$ and $\widetilde{M}$ by solving the Lyapunov equation (6). Moreover, the node subsystem (1) is determined by the dynamical equation (25), and we can choose the following simulation parameters: $R_{i}=1, C_{i}=0.1 i, I_{i}=5 \cos (t), \rho(t)=0.2$, and $Q=100 I_{10}$. Finally, initial values of states $x_{i}(0)$ and $p_{i j}(0)=p_{j i}(0)$, $i, j=1,2, \ldots, 10$, are given by randomly generating numbers in the range $(-3,3)$. The simulation results are shown in Figures 1-5 .

From Figures 1-5, we can obtain the following conclusions:

(i) From Figures 2-4, we can see that the estimation error converges asymptotically to zero. According to Definition 3, we know that the Riccati dynamical equation (13) is a state observer of subsystem (2), and the state observer is effective.

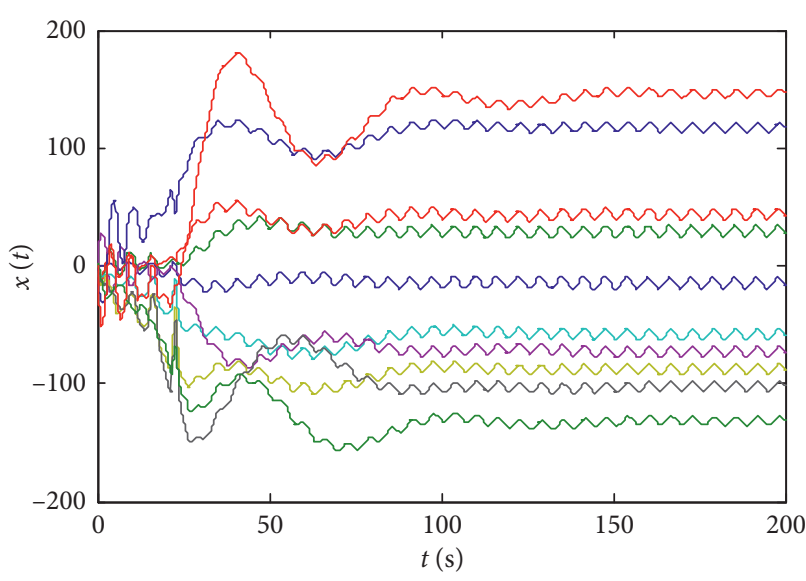

Figure 1: The state response curves of the nodes subsystem.

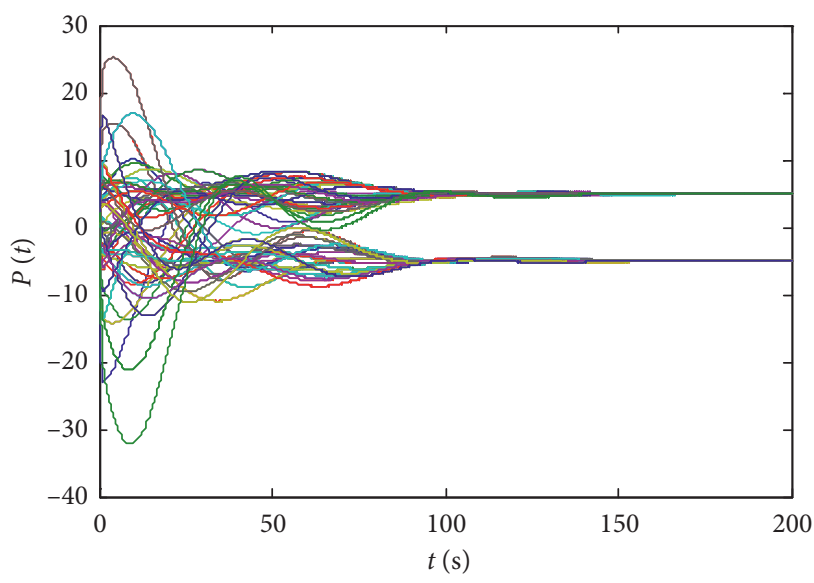

Figure 2: The response curves of the connection matrix $P(t)$.

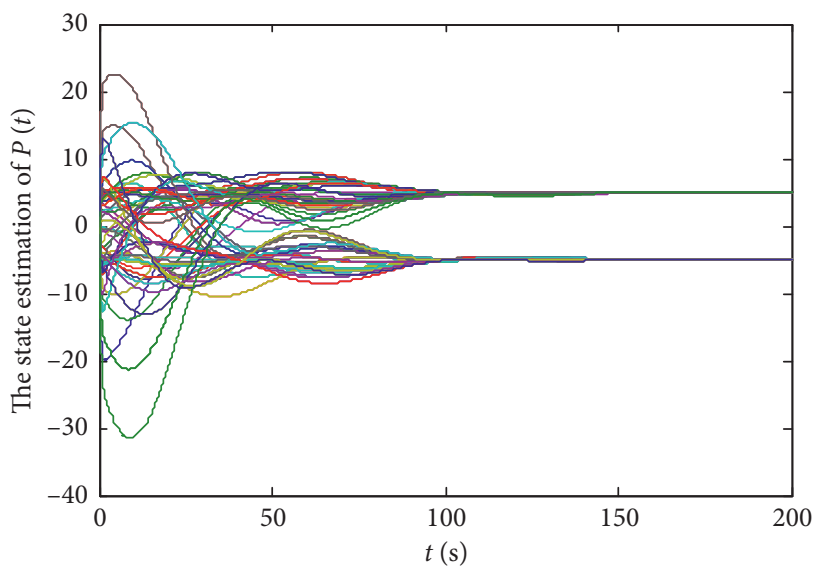

FIgURE 3: The response curves of the estimation matrix $\widehat{P}(t)$.

(ii) Figures 2 and 5 show that subsystem (2) can asymptotically track the structural balance matrix $P^{*}$, which can lead to the phenomenon of structural balance in the network. Compared with other methods of structural balance control [14, 33, 34], the biggest difference is that we can directly design 


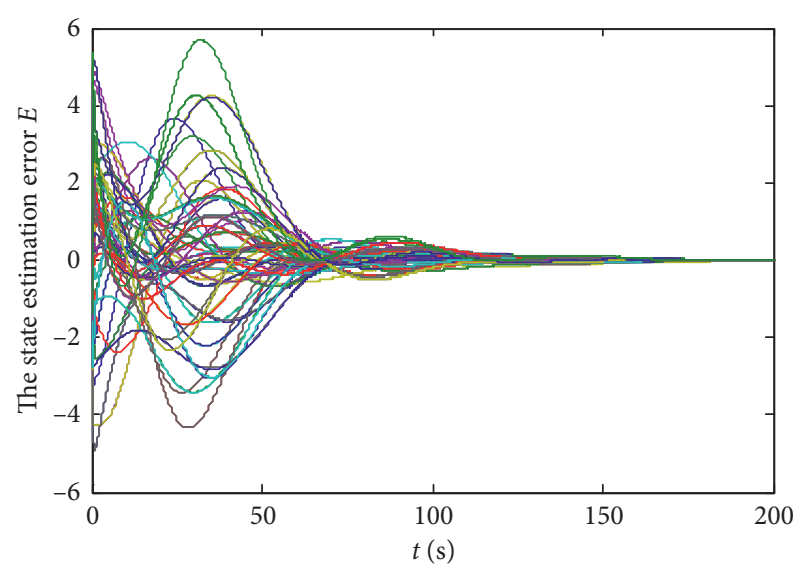

FIgURE 4: The response curves of the estimation error matrix $E$.

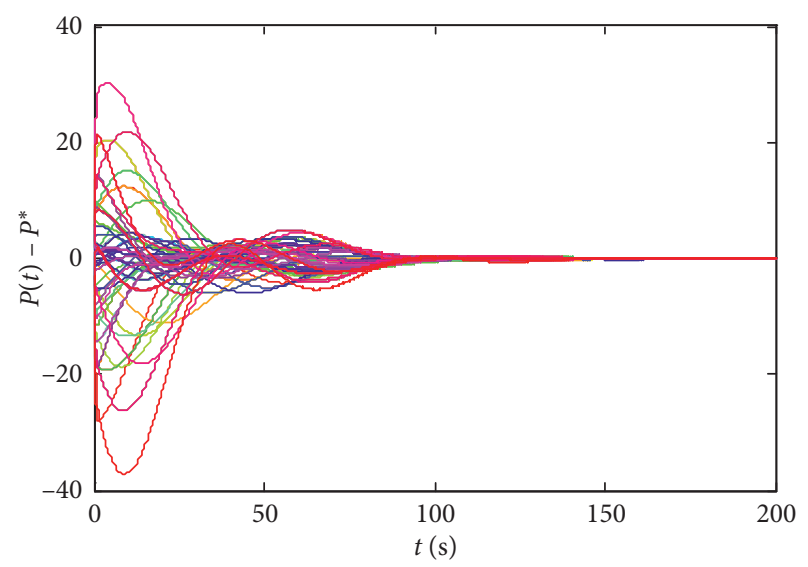

Figure 5: The response curves of the tracking error matrix $P-P^{*}$.

the structural balance controller for the connection relationship subsystem based on the estimation information of the state observer. Therefore, the controller proposed in this paper is simpler, and the requirements of form about the coupling $G(x)$ in subsystem (2) are lower.

(iii) From Figures 1 and 2, it is easy to see that the neuron (nodes) subsystem (25) and the connection relationship subsystem (2) are mutually coupled. It is worth noting that the states of the nodes are used in state observer (13) and controller (18), and it implies that the dynamic changes of the nodes can affect the observer's estimated error and the structural balance of the network. On the contrary, the dynamic changes of the connection relationships will also cause the states of nodes to be changed via the effective coupling.

\section{Conclusion}

In this paper, we have proposed a complex dynamical network model, which is composed of the node subsystem and the connection relationship subsystem, and the two subsystems are coupled with each other. Different from the existing research achievements on the state estimation of the node subsystem, we mainly focus on the state estimation of the connection relationship subsystem with outputs and design a state observer to estimate the state of the connection relationships subsystem in this paper. This encouraged us to directly design a structural balance controller for the connection relationship subsystem by using the state estimation information of the connection relationships. Based on this, the structural balance controller proposed in this paper is simpler than the existing methods of structural balance control. Therefore, the design methods of state estimation and structural balance control proposed in this paper can enrich the research results about the state estimation and structural balance of complex dynamical networks.

\section{Data Availability}

The emulation program used to support the findings of this study is included within the supplementary information file.

\section{Conflicts of Interest}

The authors declare that they have no conflicts of interest.

\section{Acknowledgments}

This work was supported by the National Science Foundation of China (61673120 and 61273219), Scientific and Technological Research Program of Chongqing Municipal Education Commission (KJ1710244, KJQN201801215, and KJ1710241), and Key Laboratory of Chongqing Municipal Institutions of Higher Education ([2017]3).

\section{Supplementary Materials}

Emulation program (Supplementary Materials)

\section{References}

[1] C. L. P. Chen, J. Wang, C.-H. Wang, and L. Chen, "A new learning algorithm for a fully connected neuro-fuzzy inference system," IEEE Transactions on Neural Networks and Learning Systems, vol. 25, no. 10, pp. 1741-1757, 2014.

[2] F. Hassanibesheli, L. Hedayatifar, P. Gawroński, M. Stojkow, D. Żuchowska-Skiba, and K. Kułakowski, "Gain and loss of esteem, direct reciprocity and Heider balance," Physica A: Statistical Mechanics and Its Applications, vol. 468, pp. 334339, 2017.

[3] H. Kim, H. Lee, M. Ahn, H.-B. Kong, and I. Lee, "Joint subcarrier and power allocation methods in full duplex wireless powered communication networks for OFDM systems," IEEE Transactions on Wireless Communications, vol. 15, no. 7, pp. 4745-4753, 2016.

[4] L. Tan, Z. Zhu, F. Ge, and N. Xiong, "Utility maximization resource allocation in wireless networks: methods and algorithms," IEEE Transactions on Systems, Man, and Cybernetics: Systems, vol. 45, no. 7, pp. 1018-1034, 2015.

[5] A. Villani, A. Frigessi, F. Liljeros, M. K. Nordvik, and B. F. de Blasio, "A characterization of Internet dating network structures among nordic men who have sex with men," PLoS One, vol. 7, no. 7, Article ID e39717, 2012. 
[6] X. Yang, J. Lam, D. W. C. Ho, and Z. Feng, "Fixed-time synchronization of complex networks with impulsive effects via non-chattering control," IEEE Transactions on Automatic Control, vol. 62, no. 11, pp. 5511-5521, 2017.

[7] L. Zhang, Y. Wang, Y. Huang, and X. Chen, "Delay-dependent synchronization for non-diffusively coupled timevarying complex dynamical networks," Applied Mathematics and Computation, vol. 259, pp. 510-522, 2015.

[8] R. Zhang, D. Zeng, S. Zhong, and Y. Yu, "Event-triggered sampling control for stability and stabilization of memristive neural networks with communication delays," Applied Mathematics and Computation, vol. 310, pp. 57-74, 2017.

[9] H. X. Hu, Q. Zhou, G. H. Wen, W. Yu, and W. Kong, "Robust distributed stabilization of heterogeneous agents over cooperation-competition networks," IEEE Transactions on Circuits and Systems II: Express Briefs, 2019, in press.

[10] J. Ma, H. Ji, D. Sun, and G. Feng, "An approach to quantized consensus of continuous-time linear multi-agent systems," Automatica, vol. 91, pp. 98-104, 2018.

[11] H.-x. Hu, W. Yu, G. Wen, Q. Xuan, and J. Cao, "Reverse group consensus of multi-agent systems in the cooperation-competition network," IEEE Transactions on Circuits and Systems I: Regular Papers, vol. 63, no. 11, pp. 2036-2047, 2016.

[12] H.-X. Hu, G. Wen, W. Yu, Q. Xuan, and G. Chen, "Swarming behavior of multiple Euler-Lagrange systems with cooperation-competition interactions: an auxiliary system approach," IEEE Transactions on Neural Networks and Learning Systems, vol. 29, no. 11, pp. 5726-5737, 2018.

[13] S. A. Marvel, J. Kleinberg, R. D. Kleinberg, and S. H. Strogatz, "Continuous-time model of structural balance," Proceedings of the National Academy of Sciences, vol. 108, no. 5, pp. 1771-1776, 2011.

[14] Z. Gao, Y. Wang, and L. Zhang, "Adaptive control of structural balance for complex dynamical networks based on dynamic coupling of nodes," International Journal of Modern Physics B, vol. 32, no. 4, Article ID 1850042, 2018.

[15] S. Wongkaew, M. Caponigro, K. Kulakowski, and A. Borzi, "On the control of the Heider balance model," The European Physical Journal Special Topics, vol. 224, no. 17-18, pp. 3325-3342, 2015.

[16] X. Wu, G. P. Jiang, and X. W. Wang, "State estimation for general complex dynamical networks with packet loss," IEEE Transactions on Circuits and Systems II: Express Briefs, vol. 65, no. 11, pp. 1753-1757, 2018.

[17] H. Li, Z. Ning, Y. Yin, and Y. Tang, "Synchronization and state estimation for singular complex dynamical networks with time-varying delays," Communications in Nonlinear Science and Numerical Simulation, vol. 18, no. 1, pp. 194-208, 2013.

[18] Z. Lei, Z. Wang, H. Gao, and X. Liu, "State estimation for discrete-time dynamical networks with time-varying delays and stochastic disturbances under the round-robin protocol," IEEE Transactions on Neural Networks and Learning Systems, vol. 28, no. 5, pp. 1139-1151, 2017.

[19] W. Li, J. Sun, Y. Jia, J. Du, and X. Fu, "Variance-constrained state estimation for nonlinear complex networks with uncertain coupling strength," Digital Signal Processing, vol. 67, pp. 107-115, 2017.

[20] R. Sakthivel, M. Sathishkumar, B. Kaviarasan, and S. Marshal Anthoni, "Synchronization and state estimation for stochastic complex networks with uncertain inner coupling," Neurocomputing, vol. 238, pp. 44-55, 2017.

[21] C.-X. Fan, F. Yang, and Y. Zhou, "State estimation for coupled output discrete-time complex network with stochastic measurements and different inner coupling matrices,"
International Journal of Control, Automation and Systems, vol. 10, no. 3, pp. 498-505, 2012.

[22] J. Hu, Z. Wang, S. Liu, and H. Gao, "A variance-constrained approach to recursive state estimation for time-varying complex networks with missing measurements," Automatica, vol. 64, pp. 155-162, 2016.

[23] C. Ma, J. Cao, L. Yang, J. Ma, and Y. He, "Effective social relationship measurement based on user trajectory analysis," Journal of Ambient Intelligence and Humanized Computing, vol. 5, no. 1, pp. 39-50, 2014.

[24] F. Zeng, N. Zhao, and W. Li, "Effective social relationship measurement and cluster based routing in mobile opportunistic networks," Sensors, vol. 17, no. 5, p. 1109, 2017.

[25] Z.-l. Gao, Y.-h. Wang, J. Xiong, L.-1. Zhang, and W.-l. Wang, "Robust state observer design for dynamic connection relationships in complex dynamical networks," International Journal of Control, Automation and Systems, vol. 17, no. 2, pp. 336-344, 2019.

[26] T. Antal, P. L. Krapivsky, and S. Redner, "Social balance on networks: the dynamics of friendship and enmity," Physica D: Nonlinear Phenomena, vol. 224, no. 1-2, pp. 130-136, 2006.

[27] P. Pattison and G. Robins, "Neighborhood-based models for social networks," Sociological Methodology, vol. 32, no. 1, pp. 301-337, 2002.

[28] T. Antal, P. L. Krapivsky, and S. Redner, "Dynamics of social balance on networks," Physical Review E, vol. 72, no. 3, Article ID 036121, 2005.

[29] K. Kułakowski, P. Gawroński, and P. Gronek, "The Heider balance: a continuous approach," International Journal of Modern Physics C, vol. 16, no. 5, pp. 707-716, 2005.

[30] V. A. Traag, P. V. Dooren, and P. D. Leenheer, "Dynamical models explaining social balance and evolution of cooperation," PLoS One, vol. 8, no. 4, Article ID e60063, 2013.

[31] S. Wongkaew, M. Caponigro, and A. Borzi, "On the control through leadership of the Hegselmann-Krause opinion formation model," Mathematical Models and Methods in Applied Sciences, vol. 25, no. 3, pp. 565-585, 2015.

[32] M. J. Krawczyk, M. del Castillo-Mussot, E. HernándezRamírez, G. G. Naumis, and K. Kułakowski, "Heider balance, asymmetric ties, and gender segregation," Physica A: Statistical Mechanics and Its Applications, vol. 439, pp. 66-74, 2015.

[33] Z. L. Gao and Y. H. Wang, "The structural balance analysis of complex dynamical networks based on nodes' dynamical couplings," PLoS One, vol. 13, no. 1, Article ID e0191941, 2018.

[34] Z. Gao, Y. Wang, L. Zhang, Y. Huang, and W. Wang, "The dynamic behaviors of nodes driving the structural balance for complex dynamical networks via adaptive decentralized control," International Journal of Modern Physics B, vol. 32, no. 24, Article ID 1850267, 2018.

[35] A. Diamandescu, " $\Psi$-bounded solutions for a Lyapunov matrix differential equation," Electronic Journal of Qualitative Theory of Differential Equations, vol. 2009, no. 17, pp. 1-11, 2009.

[36] J. Hopfield and D. Tank, "Computing with neural circuits: a model," Science, vol. 233, no. 4764, pp. 625-633, 1986. 